\title{
Electrons Extraction from MWCNT/PEO Composites
}

\author{
N. Benkara Mohammed ${ }^{1,2,}$, R. Md Nor ${ }^{1}$, N. Benouattas ${ }^{3}$ \\ ${ }^{1}$ Department of Physics, Faculty of Science, University of Malaya, 50603 Kuala Lumpur, Malaysia \\ 2 Department of Sciences and Techniques, Faculty of Sciences and Technology, University of Mohamed Bachir El- \\ Ibrahimi, Bordj-Bou-Arreridj 34000, Algeria \\ ${ }_{3}$ Laboratory of Surfaces and Interfaces of Solid Materials, University of Mohamed El-Bachir El-Ibrahimi, \\ Setif I 19000, Algeria
}

(Received 14 March 2018; revised manuscript received 15 August 2018; published online 25 August 2018)

\begin{abstract}
Electron field emission was investigated from multi-walled carbon nanotubes (MWCNT)/polyethylene oxide (PEO) composites with (5, 15, 25, 33, 40 and 50wt.\% of MWCNTs). The resulted samples were characterized using Raman spectroscopy to estimate The MWCNTs-PEO (Nanotubes-Polymer) interaction through the shifting or width alteration of the peaks. The SEM micrographs were issued to investigate the surface morphology of the samples. In addition, and in order to gain additional understanding of the filler content and the samples thermal stability; the TGA technique was applied. The current density versus applied electric field $\mathrm{J}(\mathrm{E})$ and current stability of the prepared composites were measured using a range of voltages. Here, the $33 \mathrm{wt} . \%$ composite displayed the lowest turn on field of $6.51 \mathrm{~V} / \mu \mathrm{m}$, whereas the highest current density achieved was $1.17 \mathrm{~mA} / \mathrm{cm}^{2}$ by the $40 \mathrm{wt} . \%$ composite.
\end{abstract}

Keywords: MWCNTs, PEO, Nanomposites, Field electron emission.

DOI: $10.21272 /$ jnep.10(4).04006

PACS number: 78.67.Sc

\section{INTRODUCTION}

In order to develop a novel, strong, light, conductive, smart and multifunctional composite materials, polymer matrices have been reinforced with carbon nanotubes. These tubes have exhibited excellent field emission features, a low threshold field, and a high current density [1]. On the other hand, and in order to exploit the flexibility of polymers, earlier studies have focused on integrating CNT emitters into flexible devices [2-4]. Hence, the objective of this study is to investigate field electron emission of MWCNTs embedded in polyethylene oxide thermoplastic matrix, the loading of MWCNTs is between 5 and 50 wt.\%. PEO is chosen, as it is miscible with water in all ratios due to hydration of its ether oxygen [5]. To ensure MWCNTs dispersion; the tubes were non-covalently functionalized with sodium dodecyl sulfate (SDS) anionic surfactant. In this work, ultrasonic dispersion technique was applied, because wrapping the nanotubes with SDS, guarantees that the tubes previously separated by ultrasonication will not rejoin [6].

\section{EXPERIMENTAL SET-UP}

MWCNTs used as received, they were of $95 \%$ purity, > $50 \mathrm{~nm}$ diameter and lengths between 10 and $15 \mu \mathrm{m}$, purchased from Chengdu Organic Chemicals Limited, China. MWCNT/PEO composites were fabricated by solution casting method. To obtain stable suspension; MWCNTs were ultrasonically dispersed using sodium dodecyl sulfate as surfactant; typically $1 \mathrm{~g}$ MWCNTs and 1g SDS were dissolved in $100 \mathrm{ml}$ of deionized (DI) water, the solution was ultrasonicated for 2 hours at $25 \mathrm{~W}$. To fabricate composites; $1 \mathrm{~g}$ PEO was thoroughly dissolved in $50 \mathrm{ml}$ DI water and mixed with solutions having different MWCNTs weights, making six mixtures containing between 5 and 50 wt.\% of MWCNTs. Each mixture was thoroughly mixed and subjected to ultrasonic agitation for 2 hours.

The produced liquid composites were poured in Petri dishes and left for several days to dry in air and room temperature. Dried thick films of about $60 \mu \mathrm{m}$ thickness were cut in rectangular shapes with surface areas between 0.13 and $0.32 \mathrm{~cm}^{2}$, then pasted on polished N-type Si (100) substrates. The composite films were analyzed using scanning electron microscopy (SEM), Raman spectroscopy at $532 \mathrm{~nm}$ and thermogravimetry. Field electron emission measurements were conducted at vacuum level of about $1 \cdot 10^{-7}$ mbar, utilizing a source meter unit connected to a computer. The I-V characteristics were obtained at bias voltages up to $1.1 \mathrm{kV}$ and stability curves were measured for about 34 minutes at $600 \mathrm{~V}$.

\section{RESULTS AND DISCUSSION}

Figure 1 shows raman spectra of MWCNT/PEO thick film composites before and after electrons extraction (Figure 1(a),(b)). In Figure 1(a), the uppermost plot is obtained from the pure MWCNTs. The second trace shows the spectrum obtained from composite sample with 50wt.\% MWCNTs loading. The $\mathrm{D}, \mathrm{G}$ and $\mathrm{G}$ ' peaks are present in addition to $\mathrm{C}-\mathrm{H}$ stretching bands of $\mathrm{PEO}$, the latest bands intensities are much lower than the G' band intensity. The third trace shows that $40 \mathrm{wt} . \%$ sample exhibits the same features as $50 \mathrm{wt} . \%$ with higher intensities in $\mathrm{C}-\mathrm{H}$ stretching bands. The same thing can be said for 33, 15 and $5 \mathrm{wt} . \%$, where the $\mathrm{C}-\mathrm{H}$ bands increase in intensities compared to G' whenever the carbon portion decreases, which implies that a well dispersion of the nanotubes within the polymer matrix was achieved. PEO skeletal vibrations between 400 and $1500 \mathrm{~cm}^{-1}$ and far infrared region below $400 \mathrm{~cm}^{-1}$ appear clearly

\footnotetext{
*nadjima2004@yahoo.com
} 
in the low loading samples, with reduced intensities when the MWCNTs fractions augment.
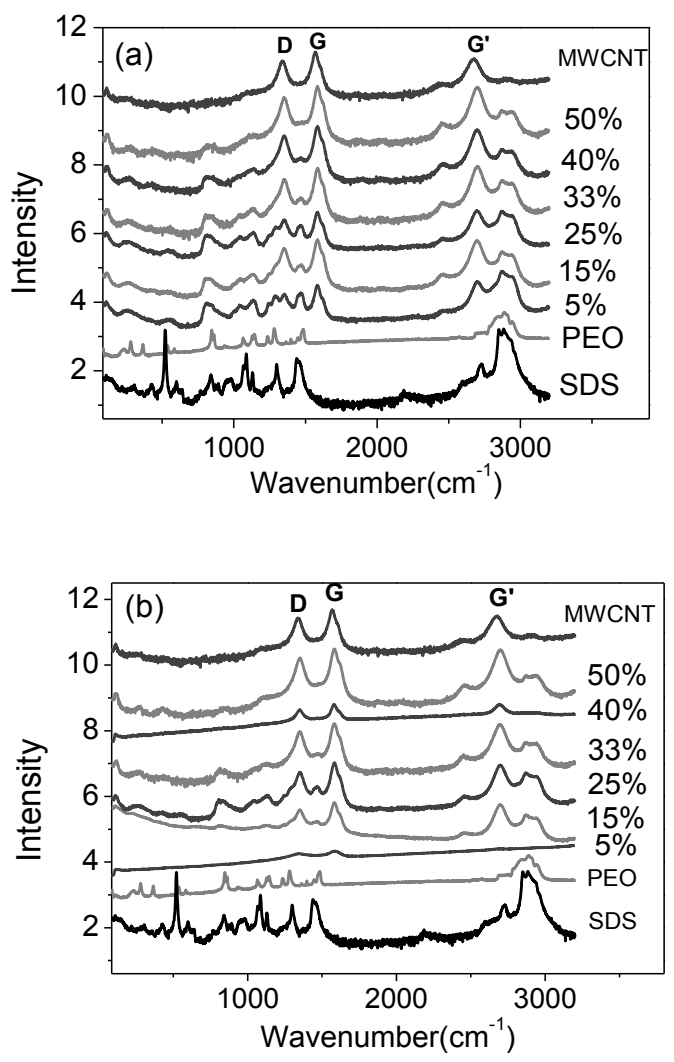

Fig. 1 - Raman spectroscopy of MWCNT/PEO thick films (a) before FEE measurements and (b) after FEE measurements

The $D$ and $G$ peaks have up-shifted in all prepared films; the G peak shifting was as high as $16.66 \mathrm{~cm}^{-1}$ in 40 and 50 wt.\% composites. The D and G shifting to upper frequencies was probably on one hand due to the nanotubes dispersion and disentanglement and on the other hand to the compression force exerted by the polymer molecules on the carbon tubes walls, as it was reported by McNally et al. [6] in a previous study but using MWCNT/PE composite.

After FEE measurement (Figure 1(b)), the same band features with slight differences were obtained. However, the spectra appear smoother than before FEE measurement, particularly in 5 and $40 \mathrm{wt} . \%$ composites. Here, contrary to the before FEE measurement case, the G-band down shifted in 40 and 50 wt.\% and reached $8 \mathrm{~cm}^{-1}$ in the two samples. Whereas D-band showed a down shift in four out of the six samples $(5,15,25$ and 33 wt.\%) and no change in two (40, 50 wt.\%). This D and $\mathrm{G}$ bands down shifting infers that less compressive force is exerted on the carbon tubes after exposure to FEE measurement. We relate it to the evaporation of the cathode material (prepared composites) to the anode during exposure to high voltage and low pressure. In fact, Purcell et al.[7] have reported that the temperature at the tip of a single MWCNT could go up to 2000K. In addition, PEO polymer holds a low melting point of 57$73^{\circ} \mathrm{C}$ [5]. The mechanical stress demonstrates its influence on the FEE results.

The intensity ratio $I_{D} / I_{G}$ suggests that almost all the emitters exhibitted higher disorder after FEE measurement. For instance; 5 and 40 wt.\% samples show that their $I_{D} / I_{G}$ increased from 0.56 to 0.87 and from 0.52 to 0.64 respectively. This augmentation signifies the increment of $s p^{3}$ hybridized carbon and specifically represents damage and defect sites in the tubes resulted from high voltage and localized heat.

Figure 2 (a-f) shows the SEM micrographs of 5 to 50 wt.\% MWCNT/PEO composites respectively. $5 \mathrm{wt} . \%$ sample exhibits a smooth surface containing pores of about $8 \mu \mathrm{m}$ length and $2 \mu \mathrm{m}$ width. The size of these pores diminishes gradually until they disappear for $50 \mathrm{wt} \%$, leading to rougher and rougher surfaces having more compacted nanotubes with nano and micro protrusions that formed after MWCNTs addition. It is noted that these protrusions are necessary for local electric field intensification in the electron field emission [8, 9].

Extremely, in Figure 2(f) corresponding to 50 wt.\% sample, some MWCNTs agglomerates are seen in the matrix, usually resulting from the Van der Waals interaction. Apparently, in 50 wt.\% the CNTs could not be wrapped perfectly, totally due to the high quantity of MWCNTs. Generally, the SEM micrographs indicate that a well and homogeneous dispersion was achieved.

Figure 3 shows TGA curves of the pure PEO and MWCNT/PEO composites. PEO started an abrupt degradation at about $335{ }^{\circ} \mathrm{C}$ and lost $95.5 \mathrm{wt} . \%$ of its weight at $401{ }^{\circ} \mathrm{C}$, the DTG curve displayed a sharp intensive and symmetric peak at $346^{\circ} \mathrm{C}$. Thus, the decomposition of the polymer PEO appeared in only one stage, whereas all the prepared composite films showed two stages decomposition.

We remarked that the first stage was in a long interval of time with a slow process of weight loss. The DTG of this first transition yielded more than one peak for all samples, except for $5 \mathrm{wt} \%$ which resembles the DTG of $\mathrm{PEO}$ as the MWCNTs portion is very small. The presence of more than one peak in the DTG curve indicates the presence of multiple components which confirms the occurrence of different carbonaceous structures in the samples. This is due to the interaction between the MWCNTs and the polymer chains which caused changes in MWCNTs C-C bonds. This result is emphasized by the Raman spectroscopy, which showed relatively high $I_{D} / I_{G}$ ratio. It is reported that changes in $\mathrm{C}$ - $\mathrm{C}$ bonding indicate the adhesion of PEO chains to MWCNTs sidewalls [10]. The second stage of TGA decomposition displayed at 309$411^{\circ} \mathrm{C}$, was abrupt and steep and is due to breaking of the main chain of PEO in the composite.

The current density-electrical field $J(E)$ curves from MWCNT/PEO cold cathodes (Figure 4), were modeled according to Fowler-Nordheim theory [9, 11].

$$
J(E)=\frac{A \beta^{2} E^{2}}{\Phi} \exp \left(-\frac{B \Phi^{3 / 2}}{\beta E}\right),
$$

where $A=1.56 \cdot 10^{-6} \mathrm{AeVV}^{-2}, B=6.83 \cdot 10^{7} \mathrm{eV}^{-3 / 2} \mathrm{Vcm}^{-1}, \Phi$ is the work function, taken to be equal to that of graphite $(5 \mathrm{eV})$ and $\beta$ is the field enhancement factor. The known linear Fowler-Nordheim plot is as follows:

$$
\ln \left(\frac{J}{E^{2}}\right)=\frac{A \beta^{2}}{\Phi}-\frac{B \Phi^{3 / 2}}{\beta E},
$$




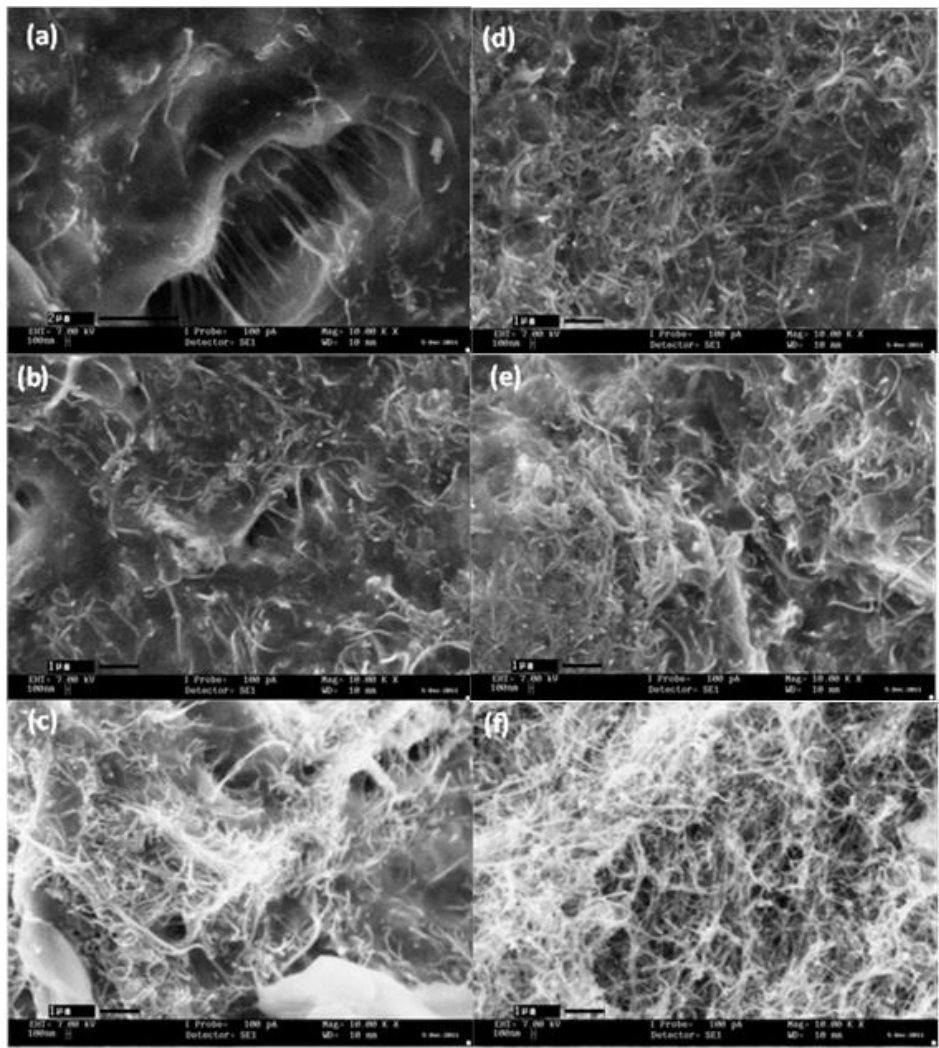

Fig. 2 - SEM micrographs of the six MWCNT/PEO thick films composites (a) 5 wt.\%, (b) 15 wt.\%, (c) 25 wt.\%, (d) 33 wt.\%, (e) 40 wt.\% and (f) 50 wt.\%

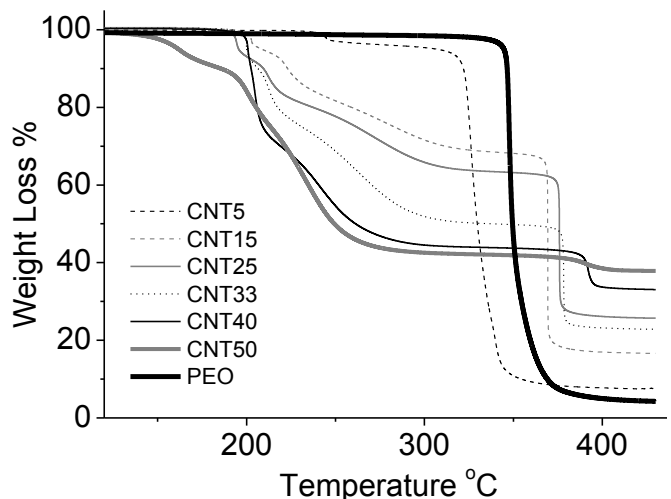

Fig. 3-TGA of pure PEO and 5 wt.\%, 15 wt.\%, 25 wt.\%, 33 wt. $\%, 40$ wt. $\%$ and 50 wt.\% MWCNT/PEO composites

where, linear fitting of equation (2) would obtain the field enhancement factor $\beta$. The approximately linear attitude of the Fowler-Nordheim (FN) (Figure 4(b)) suggests that the yielded current is produced by the field electron emission mechanisms.

The turn-on field $\left(E_{t o}\right)$, threshold field $\left(E_{t h}\right)$ and the field enhancement factor $(\beta)$; are useful parameters, which are practical for comparison with other emitters. Hence, the values of $E_{t o}$ (the applied field to produce a current density equal to $10 \mu \mathrm{Acm}^{-2}$ ), the $E_{t h}$ (the applied field at an emission current density of $0.1 \mathrm{mAcm}^{-2}$ ) and the $\beta$ for each film, deduced from current densityelectrical field curves, are drawn in Figure 5. It can be seen that the 33 wt.\% composite displayed the lowest turn on field of $6.51 \mathrm{~V} / \mu \mathrm{m}$, whereas the highest
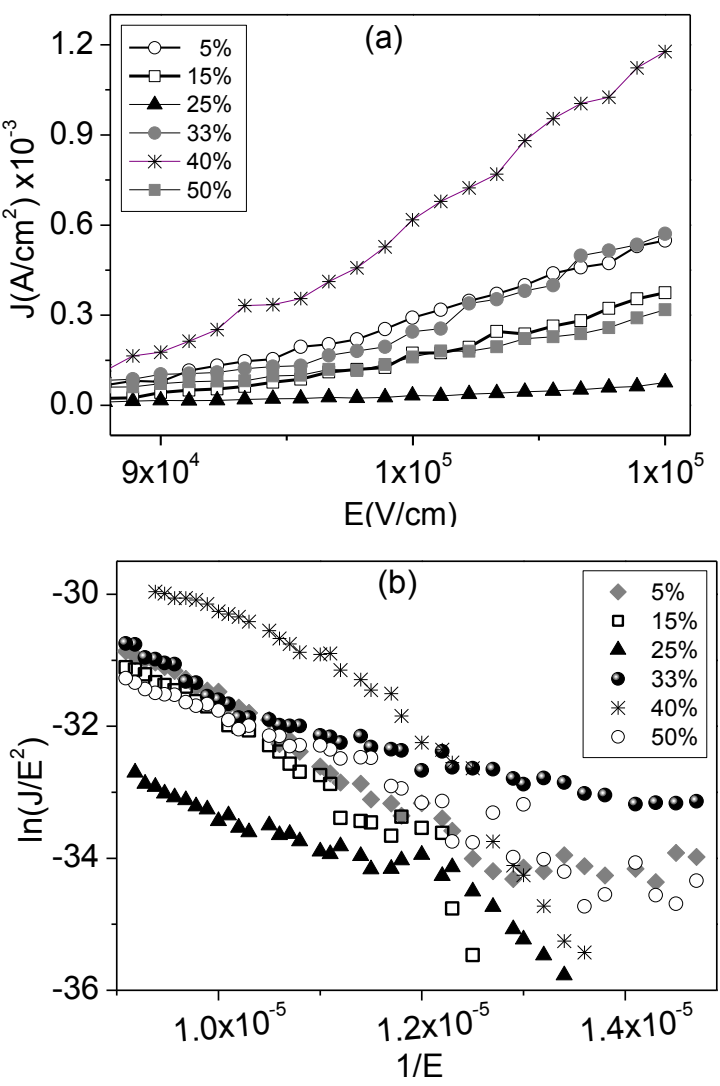

Fig. 4 - (a) Current density versus the applied electric field and (b) Fowler-Nordheim plot of the films with different MWCNTs weight concentrations 


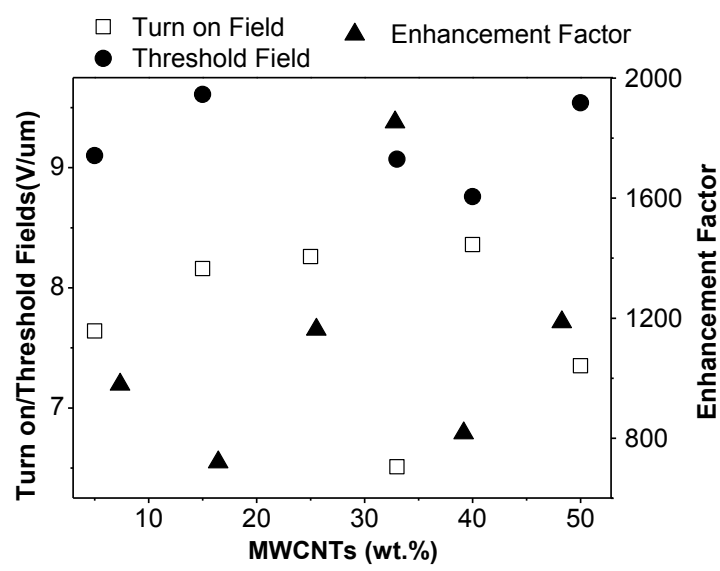

Fig. 5 - Turn on field, threshold field and enhancement factors for the different MWCNTs wt.\%.

current density was $1.17 \mathrm{~mA} / \mathrm{cm}^{2}$, achieved by the 40 wt.\% composite, which showed 8.36 and $8.76 \mathrm{~V} / \mu \mathrm{m}$ turn on and threshold fields respectively. To our knowledge, field electron emission from MWCNTs/PEO thick films $(60 \mu \mathrm{m})$ has not been investigated so far. However, regarding just MWCNTs, Lyth et al. [3] have investigated the FEE of acid oxidized MWCNTs deposited by means of dip coating on paper substrates, the $E_{t h}$ ranged between 0.8 and $11.6 \mathrm{~V} / \mu \mathrm{m}$. Where, the $E_{t h}$ is defined as the applied field at an emission current of $1 \mathrm{nA}$.

Obviously, always regarding Figure 5, there is no linear relationship between the MWCNTs loading and field emission characteristics, as was observed in earlier studies regarding other polymer composites [12,13]. Since the CNTs are homogeneously dispersed, the nanotubes/polymer interface is strong enough to effectively enhance the electrical, thermal and mechanical properties of the composite [14]. However, when nanotubes density increases, the electric field on a singular nanotube become screened by the CNTs in the vicinity [15]. Nilsson et al. found out that highly packed CNT films did not provide the best FEE results [16].

The stability of our samples was tested by current intensity measurements while maintaining constant voltage for $2000 \mathrm{~s}$ (Figure 6). Generally, all the samples exhibited a satisfaction in stability.

Samples with 25 wt.\% and 33 wt.\% showed a great stability for the whole period. While, after $420 \mathrm{~s} ; 5$ and 15 wt.\% maintained a significant stability. The current of 40 and especially $50 \mathrm{wt} . \%$ had dramatic up and down fluctuations. The normal trend in current decreasing

\section{REFERENCES}

1. S.D. Liang, L. Chen, Phys. Rev. Lett. 101, 027602 (2008).

2. Y.J. Jung, S. Kar, S. Talapatra, C. Soldano, G. Viswanathan, X. Li, Z. Yao, F.S. Ou, A. Avadhanula, R. Vajtai, S. Curran, O. Nalamasu, P.M. Ajayan, Nano Lett. 6, 413 (2006).

3. S.M. Lyth, S.R.P. Silva, Appl. Phys. Lett. 90, 173124 (2007).

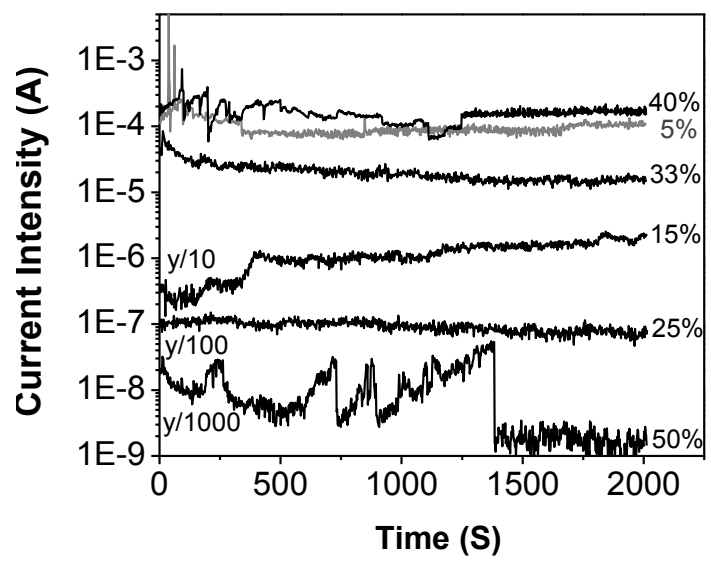

Fig 6 - Current stability in about 2000s period time for thick films with different WCNTs weight concentrations

and some fluctuations; is probably the result of dynamic rearrangement of emission sites. The local Joule heating and the large electrostatic force have their influence on the films. In deed, the substantial electrostatic force can even peel the CNTs from the substrate and deteriorate the film's performance [17].

\section{CONCLUSIONS}

MWCNT/PEO composite thick films were prepared by the ultrasonication technique. The SEM showed roughness and protrusions resulted from MWCNTs addition and played the key role in electrons emission. TGA and Raman spectroscopy emphasized the good adhesion of the polymer chains to the nanotubes walls. The thermal stability of PEO was improved by the incorporation of MWCNTs in the polymer matrix. Regarding field electron emission, Regarding field electron emission; the best enhancement factor (B) and the lowest turn on field were 1853 and $6.51 \mathrm{~V} / \mu \mathrm{m}$ respectively, achieved by 33wt.\% emitter. The highest current density $\left(1.17 \mathrm{~mA} / \mathrm{cm}^{2}\right)$ was produced by $40 \mathrm{wt} . \%$ composite. The MWCNT/PEO thick films emitters presented a high degree of stability during $34 \mathrm{~min}$ emission, except for 50wt.\% which had a dramatic up and down fluctuations, most obviously at about 1400s. In this view, we are confident that the devices based on MWCNT/PEO will show enhancements in the FEE field.

\section{ACKNOWLEDGEMENT}

This work is funded by The Ministry of Science and Innovation of Malaysia under the FRGS grant No. FP008/2010B.
4. H.S. Sim, S.P. Lau, H.Y. Yang, L.K. Ang, M. Tanemura, K. Yamaguchi, Appl. Phys. Lett. 90, 143103 (2007).

5. M.M. Crowley, F. Zhang, J.J. Koleng, J.W. McGinity, Biomaterials 23, 4241 (2002).

6. T. McNally, P. Postschke, P. Halley, M. Murphy, D. Martin, S.E.J. Bell, G.P. Brennan, D. Bein, P. Lemoine, J.P. Quinn, Polymer 46, 8222 (2005). 
7. S. Purcell, P. Vincent, Phys. Rev. Lett. 88, 105502 (2002).

8. O. Gröning, O.M. Küttel, P. Gröning, L. Schlapbach, J. Vac. Sci. Technol. B 17, 1970 (1999).

9. J.M. Bonard, J.P. Salvetat, T. Stockli, W.A. de Heer, L. Forro, A. Chatelain, Appl. Phys. Lett. 73, 918 (1998).

10. Y.L. Liu, Y.H. Chang, M. Liang, Polymer 49, 5405 (2008).

11. R.B. Rakhi, K. Sethupathi, S. Ramaprabhu, Appl. Surf. Sci. 254, 6770 (2008).

12. H. Jung, S.Y. An, D.M. Jang, J.M. Kim, J.Y. Park, D. Kim, Carbon 50, 987 (2012).

13. R.C. Smith, J.D. Carey, R.J. Murphy, W.J. Blau,
J.N. Coleman, S.R.P. Silva, Appl. Phys. Lett. 87, 263105 (2005).

14. Z. Xu, Y. Niu, L. Yang, W. Xie, H. Li, Z. Gan, Polymer 51, 730 (2010)

15. Y. Li, Y. Sun, J.T.W. Yeow, Nanotechnology 26, 242001 (2015).

16. L. Nilsson, O. Groening, C. Emmenegger, O. Kuettel, E. Schaller, L. Schlapbach, H. Kind, J.M. Bonard, K. Kern, Appl. Phys. Lett. 76, 2071 (2000).

17. Y. Cheng, O.Zhou, Comptes Rendus Physique 4, 1021 (2003). 